\section{Implementación del diagnóstico molecular del VPH: experiencia chilena en curso}

\author{
GINA F. MERINO ${ }^{1,7,8}$, ROBERTO A. ALTAMIRANO ${ }^{2}$, \\ JUAN HERRERA ${ }^{3}$, CLEMENTE ARAB ${ }^{4}$, DUNJA D. ROJE 5 , \\ SERGIO BECERRA ${ }^{1}$, PAULA ESCOBAR ${ }^{1,6}$, JUVENAL A. RÍOS ${ }^{1,7,8,9}$
}

\section{Molecular diagnosis of human papillomavirus infection. The Chilean experience}

Cervical Cancer ( $\mathrm{CaCu}$ ) has a heterogeneous epidemiological behavior throughout the planet, depending on regional socioeconomic development level. Some developed countries predict a potential eradication of this cancer in the next 100 years, while in Chile it still constitutes a pending challenge. Incidence rates show a slow but sustained downward prob, however, mortality has continued to fluctuate between 500-600 cases per year. A few years ago, vaccination against Human Papillomavirus (HPV), the main causal agent for this tumor, was consolidated as a public policy, both in girls and in boys. However, the technological leap in the screening prob was pending, from cytology to molecular diagnosis of the agent (HPV). In this report, we update our most recent data (2018) regarding $\mathrm{CaCu}$ mortality, then review global guidelines and experiences in HPV screening. Finally, we offer an account of the strategies that our health system is promoting to address the screening of the disease and whose molecular approach generates the widest worldwide scientific consensus. We also recognize the main barriers and future challenges, which, if overcome, would allow us to be in line with the mandate of WHO to control this women's health problema.

(Rev Med Chile 2021; 149: 1339-1346)

Key words: Alphapapillomavirus; Mass Screening; Molecular Diagnostic Techniques; Public Health; Uterine Cervical Neoplasms.
'Departamento de Manejo Integral de Cáncer y otros Tumores, Ministerio de Salud. Chile.

${ }^{2}$ Servicio de Ginecología y Obstetricia, Hospital San Borja Arriarán. Departamento de Obstetricia y Ginecología, Facultad de Medicina, Universidad de Chile. Santiago, Chile.

${ }^{3}$ Departamento de Gestión de Cuidados, División de Atención Primaria, Ministerio de Salud. Chile.

${ }^{4}$ Servicio de Ginecología y Obstetricia, Hospital Doctor Luis Tisné Brousse. Departamento de Obstetricia y Ginecología, Facultad de Medicina. Universidad de Chile. Santiago, Chile.

${ }^{5}$ Fundación Nuestros Hijos. Chile.

${ }^{6}$ Departamento de Cirugía Oriente, Facultad de Medicina, Universidad de Chile. Santiago, Chile.

${ }^{7}$ Escuela de Medicina, Facultad de Medicina y Ciencia, Universidad San Sebastián. Santiago, Chile.

${ }^{8}$ Escuela de Medicina, Facultad de Ciencias, Universidad Mayor. Santiago, Chile. 9Servicio de Anatomía Patológica, Instituto Nacional del Cáncer de Chile. Santiago, Chile.

Trabajo no recibió financiamiento. Los autores declaran no tener conflictos de interés.

Recibido el 31 de marzo de 2021, aceptado el 25 de julio de 2021.

\section{Correspondencia a:}

Juvenal A. Ríos Leal M.D., M.Sc., Ph.D. Instituto de Medicina Traslacional, Centro de Oncología de Precisión (COP), Escuela de Medicina, Facultad de Ciencias, Universidad Mayor. Santiago, Chile. jriosleal@gmail.com

\section{Consideraciones generales}

1 1 cáncer cervicouterino $(\mathrm{CaCu})$ es la tercera causa de mortalidad oncológica femenina en el mundo con más de 500.000 nuevos casos diagnosticados cada año y 270.000 muertes anuales. En Chile, al año 2018, fueron diagnosticadas alrededor de 1.500 nuevos casos, falleciendo 609, cifra que ha tendido a estabilizarse en los últimos 5 años ${ }^{1-4}$.
Durante las décadas pasadas, se han realizado múltiples esfuerzos para disminuir su incidencia y mortalidad, un ejemplo de ello fue la implementación del tamizaje con el Papanicolau (PAP), que permitió una reducción en la mortalidad cercana a $60-70 \%$ en los lugares donde se implementó un programa de tamizaje con una cobertura adecuada (se recomienda cobertura de $70-80 \%$ ). En Chile se implementó este programa a partir de 1987, como un pilar fundamental de la atención 
primaria, obteniendo reducciones en la mortalidad de alrededor de $66 \%$ en un corto período de tiempo ${ }^{5}$.

A inicios de la década 2000-2010 se pudo entender casi completamente el origen infeccioso de este particular tipo de cáncer, descubriéndose que el agente causal correspondía al virus papiloma humano (VPH), dicha gesta científica cambió la perspectiva y el enfrentamiento de esta enfermedad oncológica ${ }^{6,7}$. A partir de ese momento se iniciaron dos caminos para el control y prevención del $\mathrm{CaCu}$ en el mundo, uno a través de la creación de una vacuna, la cual permite combatir la infección y persistencia del VPH en el organismo. La otra consiste en modernizar el método para realizar el tamizaje secundario, introduciéndose una prueba molecular que nos permite identificar a las portadoras de la infección por los VPH de alto riesgo oncológico, aumentando la sensibilidad entre 30 y 40\% ${ }^{8}$. Destacando a los VPH 16 y 18, responsables de $70 \%$ de los casos de $\mathrm{CaCu}$, como también a los tipos $31,33,35,39,45,51,52,56,58$, 59 y $68^{9-12}$. La introducción de la prueba molecular del VPH nos permitirá disminuir el porcentaje de falsos negativos que presenta el tamizaje con PAP, que es alrededor de $30 \%$ en cifras nacionales e internacionales ${ }^{13-15}$. Esto significa que una de cada tres mujeres podría presentar una lesión preinvasora o invasora y no ser reconocida por este examen, atrasando su tratamiento, afectando la sobrevida y los años de vida saludables perdidos. La prueba de VPH, al detectar la presencia del virus nos permite aumentar la sensibilidad de detección (99\%) y realizar el tratamiento adecuado. Pero el valor más importante de este examen estaría en el valor predictivo negativo, ya que sabemos que se requiere de la presencia del VPH prácticamente en $100 \%$ para presentar un $\mathrm{CaCu}$ y así poder postergar el próximo tamizaje en 5 años ${ }^{16}$.

En este trabajo queremos poner en evidencia los primeros esfuerzos que el sistema de salud chileno (infraestructura, recursos humanos y aspectos económicos) está llevando a cabo, para implementar la prueba molecular del VPH, que en conjunto con la vacunación y el tratamiento oportuno de las lesiones preinvasoras e invasoras, lo que forma parte del proyecto 2020-2030 (9070-90) de la OMS, se pretende erradicar el $\mathrm{CaCu}$ del planeta, de modo que, para el año 2100 presentemos menos de 4 casos por 100.000 mujeres con $\mathrm{CaCu}^{17,18}$.

\section{Lecciones aprendidas desde la experiencia internacional}

Para esta sección, se describió la situación actual de: la Unión Europea (UE), Estados Unidos de Norteamérica (EEUU), México y Australia, siendo este último un modelo especialmente reconocido como exitoso en la implementación de la tecnología VPH, y los tres primeros, por representar regiones con una suficiente heterogeneidad en cuanto a la orgánica de sus sistemas sanitarios, el grado de participación de sus agencias de salud y las garantías que otorga el estado a la población en esta materia.

A continuación, se describen las principales características y enseñanzas de estas cuatro regiones del planeta para abordar el control del $\mathrm{CaCu}$ mediante la incorporación de la prueba de detección molecular de VPH:

\section{Unión Europea (UE)}

En esta región, cada uno de sus países con una propia "estrategia VPH", muestra una heterogeneidad en cuanto enfoque y grado de avance ${ }^{19}$. La más reciente información (2018) documenta que, en 22 de los 28 países miembros de la UE, existe al menos un cierto grado de establecimiento de esta tecnología sanitaria ${ }^{20}$. Al 2016, se reportan las siguientes modalidades de acceso poblacional: opción oportunista de tamizaje, programas piloto incipientes, implementación progresiva, programas poblacionales establecidos, mientras que otros reportan estar en transición entre una etapa y otra. En relación con el rango etario en que se aplica la prueba, la gran mayoría de la UE ha optado por el rango de 25 a los 59-69 años, otros mantienen el mismo límite inferior, sin definir una edad "techo" de tamizaje y un porcentaje minoritario no ha definido rango. Un tema de amplia discusión en la comunidad científica mundial es el intervalo de años con que se toma la prueba ${ }^{21}$, en este sentido, la gran mayoría de la UE recomienda cada 3 o 5 años y un porcentaje menor no lo tiene definido. En relación con el mecanismo de transición tecnológica desde las pruebas convencionales (citológica u otras) al enfoque molecular, la gran mayoría prefiere la prueba primaria y "triage" de VPH, es decir, la utilización de un método adicional que mantenga una alta sensibilidad, pero que contenga la derivación al nivel siguiente para procedimientos diagnósticos, para ello se puede realizar 
repetición de la prueba a los 12 meses más PAP reflejo (PAP tomado en el mismo momento que la prueba de VPH, pero leído ante el resultado de un genotipo de alto riesgo oncogénico) o efectuar la genotipificación de VPH 16 y 18 directamente $^{5}$; un porcentaje minoritario reporta el "cotest", que quiere decir PAP y prueba de VPH, con citología como punto de partida ${ }^{22}$. Cabe señalar que muchos de estos programas datan de 1960, algunos de ellos fracasaron en el proceso de transición por falta de financiamiento estatal y en otros casos exitosos, se trabajó en estrecha colaboración entre el estado, sociedades científicas, privados y pacientes.

\section{Estados Unidos de Norteamérica}

Hasta la fecha este país, no posee una estrategia nacional de tamizaje de $\mathrm{CaCu}$, sin embargo, existe un programa de detección temprana de $\mathrm{CaCu}$ y cáncer de mama (NBCCEDP), el cual cubre a menos del $25 \%$ de las mujeres del país y, además, no posee aseguramiento y cobertura financiera suficiente $^{23}$. Cabe señalar que la prueba de VPH con captura híbrida fue aprobada por la Administración de Medicamentos y Alimentos (FDA) ya en 1999, luego en 2002, el NBCCEDP la incorporó en su arsenal de servicios y al año siguiente (2003) la Sociedad Americana de Obstetricia y Ginecología en conjunto con la Sociedad Americana de Cáncer, actualizaron sus guías clínicas, recomendando el "cotest" con intervalos de 3 años y manteniendo como opción, la prueba citológica por sí sola ${ }^{24,25}$. Dadas las particularidades intrínsecas de este sistema sanitario, sumado a sus diferencias económico-estructurales en los distintos estados federados, la experiencia americana recomienda que i) se siga considerando la estrategia oportunista como una opción válida; ii) se fortalezca la investigación, innovación y difusión científica en los médicos, con objeto de generar tecnologías cada vez más económicas y accesibles a toda la población, y iii) considerando que existe una comprobada costo-efectividad a mediano y largo plazo, en relación con la prueba de VPH comparado con el PAP ${ }^{26}$, su uso debe ser impulsado con mayor vigor en aquellas regiones con menor desarrollo económico, incluso considerando la modalidad de "autotoma".

\section{México}

Introdujo por primera vez la prueba de VPH con captura híbrida en 2008 a través de un programa piloto en la zona de Morelos. El 2009 se expandió en modalidad de implementación incremental a 21 de 32 estados con 12 laboratorios regionales, priorizando municipalidades con bajo nivel socioeconómico. En el 2010 comprendió a 32 estados. Tras este proceso trasformador, el sistema de salud mexicano considera fundamental: i) sumar nuevos estados, toda vez que ninguno de los ya implementados sufra una interrupción; ii) mantener la capacitación de los equipos de salud a cargo; iii) generar flujogramas claros para operacionalizar homogéneamente la implementación; iv) asegurar el financiamiento estatal al programa, y v) centrar la estrategia en la paciente, considerando aspectos biopsicosociales, inclusive como la consejería ${ }^{23}$.

\section{Australia}

En diciembre de 2017, Australia comenzó la transición del PAP a la prueba VPH con PAP reflejo. El proceso no estuvo exento de dificultades, entre ellas destacan que, en el momento del primer lanzamiento del programa, el registro nacional de cáncer no estaba puesto a punto y tampoco se contaba con la capacidad suficiente de colposcopía para recibir adecuadamente un porcentaje de pruebas positivas. Hoy, este país se alza como una potencia líder en la materia, tanto así que proyectan lograr una erradicación casi total del $\mathrm{CaCu}$ hacia fines de $2040^{27}$. En este sentido cabe enumerar algunas de las principales enseñanzas y recomendaciones que como sistema de salud australiano consideran esencial para replicar este salto sanitario: i) impulsar una robusta campaña comunicacional a profesionales y pacientes; ii) involucrar a la sociedad civil en la toma de decisiones; iii) considerar la "autotoma" para poblaciones nunca o poco tamizadas, con especial foco en las reducciones indígenas y poblaciones de menores recursos; iv) preparar al sistema para un peak de casos nuevos, en términos de infraestructura, equipamiento y recurso humano; v) concentrar la demanda de colposcopía en ciertas regiones; vi) utilizar guías clínicas disponibles en dispositivos móviles y vii) robustecer digitalmente el registro nacional de cáncer ${ }^{28}$.

\section{Experiencia local}

En Chile, a pesar de contar con un programa nacional de detección y tratamiento del $\mathrm{CaCu}$ 
desde 1987 y de haber disminuido la tasa de mortalidad y mantenerla relativamente estable en los últimos años (Gráfico 1), la cobertura de tamizaje para esta enfermedad en los últimos 5 años promedia $58,5 \%$, sin conseguir la cobertura recomendada, que es $80 \%$ de las mujeres de 25 a 64 años con PAP vigente por 3 años.

Según la encuesta CASEN del año 2017, las principales razones por las que las mujeres no se realizaron su PAP en el transcurso de 3 años fueron que este no le corresponde, creencia de no necesitarlo, olvido de toma, falta de tiempo para acudir a un centro asistencial para su realización, y miedo o disgusto en la toma del examen.

A estos antecedentes se adicionan argumentos técnicos que detonaron la decisión de comenzar a ejecutar una estrategia de implementación de la prueba molecular de VPH en el país, estos fueron: i) evidencia consensuada en la Guía de Práctica Clínica de Cáncer Cervicouterino 20155; ii) lanzamiento del primer Plan Nacional de Cáncer en donde se prioriza y reconoce la necesidad de la incorporación de la prueba de $\mathrm{VPH}^{29}$; iii) el llamado de la Organización Mundial de la Salud (OMS) para acelerar la eliminación de esta enfermedad e implementar una serie de metas durante el período 2018-2030 que contribuyan a esta causa ${ }^{30}$ y la exitosa experiencia chilena para la detección temprana de $\mathrm{CaCu}$ con la prueba de VPH en más de 8000 mujeres de Santiago ${ }^{31}$.

A comienzos del año 2019, se inició el trabajo para incorporar la prueba de VPH en mujeres de 30 a 64 años en trece de los veintinueve servicios de salud de Chile, los cuales abarcan una población de aproximadamente 1.867.847 mujeres inscritas en los establecimientos de atención primaria y validadas por FONASA, de un total de 3.368.626 mujeres en Chile en este rango etario, por lo que se estimó que serían necesarios 90.600 test de VPH para la puesta en marcha, los cuales fueron ejecutados durante los últimos 3 meses del año 2019. Para ello fue necesario planificar, sensibilizar y reorganizar los procesos asistenciales en la red de atención.

En Chile, de manera inicial, la prueba de VPH se realizó en modalidad "cotest", debido a que el tamizaje se realiza por PAP, examen asociado a disponibilidad presupuestaria, garantizado por la Ley No 19.966 (de garantías explícitas de salud, más conocida como el GES) Durante la implementación inicial, se trabajó de manera simultánea en temas regulatorios y normativos que permitirán incluir estas pruebas dentro del arsenal para prestaciones contempladas en el tamizaje de $\mathrm{CaCu}$ en el régimen de garantías en salud. De acuerdo con esto, trece servicios de salud fueron habilitados para realizar la toma simultánea de "cotest" en la población femenina de 30 a 64 años que cumpliera con los criterios de inclusión, tales como pérdida de vigencia de PAP mayor a 3 años o nunca haberse realizado un PAP. De este modo y según el flujograma revisitado por Ferrecio en $2018^{32}$, se

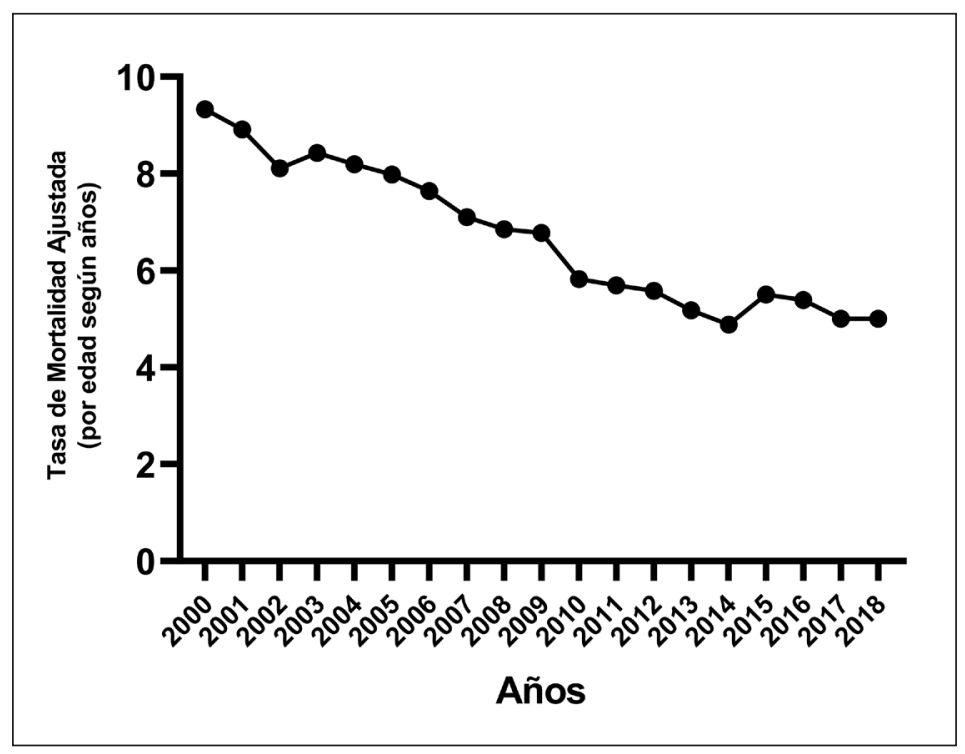

Gráfico 1. Mortalidad del cáncer cervicuterino en Chile. período 2000-2018. Elaboración propia con datos obtenidos del Departamento de Epidemiología, Ministerio de Salud, Chile. 
planificó para los próximos años la migración para implementar de toma de VPH como un método de tamizaje primario, con el consecuente procesamiento posterior del PAP, estrategia denominada PAP reflejo, el cual mejora su sensibilidad al contar con información del estatus de VPH.

Los pasos ejecutados para el éxito de esta incorporación los abordaremos en 6 dimensiones (Diagrama 1): técnico-científico, financiero, infraestructura y equipamiento, capacitaciones del personal sanitario involucrado, logística y modificaciones de los sistemas de registro. En la primera dimensión, se conformaron equipos técnicos intra y extra-Ministerio de Salud (MINSAL), compuestos principalmente por ginecólogos oncólogos, patólogos, radioterapeutas y matronas/es; simultáneamente, se desarrolló abogacía con los tomadores de decisión y solicitud de financiamiento interno en MINSAL que permitió la compra centralizada de las determinaciones y el reforzamiento del recurso humano y de equipamiento de los centros de atención primaria, unidades de patología cervical, laboratorios de anatomía patológica y laboratorios clínicos. En tercer lugar, se efectuó la habilitación de espacios físicos para la instalación de equipos de procesamiento, adquiridos en comodato, que conformaron la red de laboratorios que informaron las pruebas de VPH.
En una cuarta dimensión, se efectuaron capacitaciones presenciales y en línea a un total de 3.194 profesionales de salud. En quinto lugar, se realizó un estudio de mercado de proveedores en Chile que dispusieran de la técnica de detección molecular y se precisó si ello se ajustaba a lo requerido por MINSAL, de esta manera se adquirieron un total de 90.600 determinaciones para la detección molecular de VPH con genotipificación individualizada para los tipos 16 y 18 , y agrupada para otros genotipos de alto riesgo oncogénico. Esta logística consideró la adquisición de pruebas, reactivos, insumos, software de los equipos de procesamiento e interoperaciones con el actual sistema de registro de tamizaje que fue modificado para incorporar el resultado de esta prueba.

Durante el proceso de implementación, se incorporó esta prueba en 133 de 148 comunas de los 13 servicios de salud que participaron en esta primera etapa, lo que representó el $90 \%$ de cobertura geográfica de implementación.

\section{Conclusiones y desafíos futuros}

En este artículo pudimos examinar el momento epidemiológico que atraviesa nuestro país en relación con el control del $\mathrm{CaCu}$. Tal y como

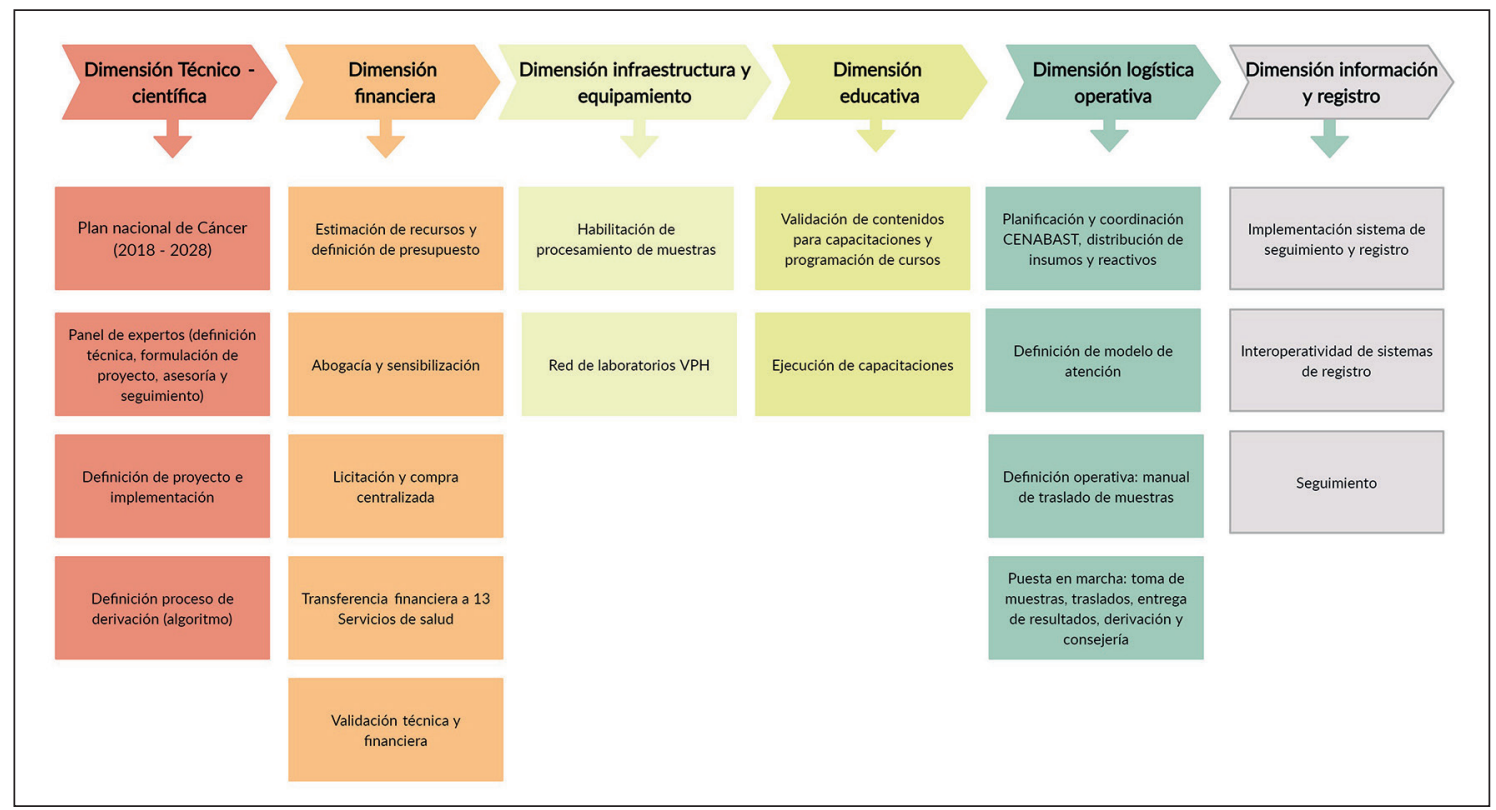

Diagrama 1. 
se comentó en la introducción, la tendencia a la estabilización de la mortalidad y no al quiebre a la baja en los casos, obligaba a dar un salto tecnológico importante, conducente a completar el arco de estrategias sanitarias que, ya hace más de una década estaban siendo indiscutiblemente recomendadas por la comunidad médica y científica internacional.

Asimismo, también tuvimos la oportunidad de resumir los procesos de implementación de la prueba molecular para la detección del VPH, bajo el prisma de cuatro disímiles experiencias mundiales en cuanto a su orgánica y recursos asociados, extraer sus lecciones aprendidas y poder replicar los ejemplos más exitosos.

Luego, nos concentramos en relatar la experiencia local, desde las dimensiones técnicas y políticas, considerando las principales barreras de un proceso que aún está en marcha y a medio camino de consolidarse. A este respecto, finalmente quisiéramos puntualizar los principales desafíos que tenemos por delante, los que deben superarse para garantizar la instalación completa de esta política pública:

1. En el ámbito financiero, se requiere actualizar el arsenal de prestaciones financiadas en la Ley $\mathrm{N}^{\circ} 19.966$ que establece un régimen de garantías explícitas en salud (GES) para el problema de $\mathrm{CaCu}$, incorporando la prueba de $\mathrm{VPH}$ como parte del tamizaje primario y la citología con PAP reflejo, a modo de garantizar este nuevo enfoque, en una glosa presupuestaria establecida año a año y sin que corra el riesgo de fracasar durante la instalación de la mejora.

2. En el ámbito de la provisión de servicios asistenciales, se debe consolidar la implementación de la prueba molecular de VPH, de manera coordinada y articulada a los "cito-laboratorios" y a las Unidades de Diagnóstico Molecular Oncológico (UDMO) asociadas a los Servicios de Anatomía Patológica. Asimismo, los datos aquí recabados (registro unificado) deberán tributar e interoperar debidamente con el Registro Nacional de Cáncer.

3. En el ámbito poblacional, el gran desafío es mejorar la cobertura del tamizaje mediante la creación de conciencia y adherencia a las acciones del programa de prevención de $\mathrm{CaCu}$ de las mujeres en el curso de vida, que consideren el autocuidado, vacunación, tamizaje, diagnóstico y tratamiento precoz.
4. Por otra parte, se requiere fortalecer la investigación en el ámbito de la evaluación permanente del impacto de la política misma, como también aquella que estimule generación de conocimiento local, esencial para la toma de decisiones de precisión en nuestra población con $\mathrm{CaCu}$.

5. Por último, es necesario trabajar continuamente en la gestión del cambio de los profesionales de la salud, quienes son los promotores y ejecutores de las acciones asociadas a la implementación de esta nueva tecnología.

Agradecimientos: Los autores agradecen a la Comisión Asesora Ministerial del Programa de Cáncer Cervicouterino, organismo multidisciplinario integrado por clínicos, epidemiólogos y científicos que han apoyado permanente el desarrollo de este programa. A la Sociedad Chilena de Obstetricia y Ginecología, Sociedad Chilena de Ginecología Oncológica y al Centro de Oncología Preventiva de la Universidad de Chile (Quinta Normal). Los autores, según corresponda, también agradecen a sus respectivas casas de estudio superiores; USS (GFM, JAR), UCHILE (PE, RA), UMAYOR (JAR, GFM), por potenciar permanentemente alianzas entre la academia y el Estado. Por último, cabe agradecer especialmente a la $E U$, Mg. Patricia Cerda, profesional del Departamento de Epidemiología, MINSAL, por suministrarnos las cifras y datos relacionados a la mortalidad del Cáncer en Chile. También el autor JAR agradece al Instituto Nacional del Cáncer de Chile, por financiar sus estudios de formación como especialista en Anatomía Patológica (en curso).

\section{Referencias}

1. Arbyn M, Weiderpass E, Bruni L, de Sanjosé S, Saraiya M, Ferlay J, et al. Estimates of incidence and mortality of cervical cancer in 2018: a worldwide analysis. Lancet Glob Heal [Internet]. 2020 Feb 1 [cited 2021 Jan 25]; 8 (2): e191-203. Available from: www.thelancet.com/ lancetgh

2. Ferlay J, Soerjomataram I, Dikshit R, Eser S, Mathers C, Rebelo M, et al. Cancer incidence and mortality worldwide: Sources, methods and major patterns in GLOBOCAN 2012. Int J Cancer [Internet]. 2015 Mar 1 [cited 2021 Jan 25]; 136 (5): E359-86. Available 
from: https://onlinelibrary.wiley.com/doi/full/10.1002/ ijc. 29210.

3. Global Cancer Observatory [Internet]. [cited 2019 Sep 18]. Available from: https://gco.iarc.fr/

4. Series y Gráficos de Mortalidad - DEIS [Internet]. [cited 2019 Jan 11]. Available from: http://www.deis.cl/ series-y-graficos-de-mortalidad/

5. Guías Clínicas AUGE Cáncer Cérvico Uterino. 2015. http://www.repositoriodigital.minsal.cl/handle/2015/534

6. Durst M, Gissmann L, Ikenberg H, Zur Hausen H. A papillomavirus DNA from a cervical carcinoma and its prevalence in cancer biopsy samples from different geographic regions. Proc Natl Acad Sci U S A [Internet]. 1983 Jun 1 [cited 2021 Jan 25]; 80 (12 I): 381-5. Available from: https://www.pnas.org/content/80/12/3812

7. Zur Hausen H. Papillomaviruses and cancer: From basic studies to clinical application [Internet]. Vol. 2, Nature Reviews Cancer. European Association for Cardio-Thoracic Surgery 2002 [cited 2021 Jan 25]; 342-50. Available from: www.nature.com/reviews/cancer

8. Herrero R, Ferreccio C, Salmerón J, Almonte M, Sánchez GI, Lazcano-Ponce E, et al. New Approaches to Cervical Cancer Screening in Latin America and the Caribbean. Vaccine [Internet]. 2008 Aug 19 [cited 2021 Jan 25]; 26 (SUPPL. 11). Available from: https://pubmed.ncbi.nlm.nih.gov/18945402/

9. De Sanjose S, Quint WGV, Alemany L, Geraets DT, Klaustermeier JE, Lloveras B, et al. Human papillomavirus genotype attribution in invasive cervical cancer: a retrospective cross-sectional worldwide study. Lancet Oncol [Internet]. 2010 Nov [cited 2021 Jan 25]; 11 (11): 1048-56. Available from: https://pubmed.ncbi.nlm.nih. gov/20952254/

10. Li N, Franceschi S, Howell-Jones R, Snijders PJF, Clifford GM. Human papillomavirus type distribution in 30,848 invasive cervical cancers worldwide: Variation by geographical region, histological type and year of publication. Int J Cancer [Internet]. 2011 Feb 15 [cited 2021 Jan 25]; 128 (4): 927-35. Available from: https:// onlinelibrary.wiley.com/doi/full/10.1002/ijc.25396

11. Ferreccio C, Van De Wyngard V, Olcay F, Domínguez MA, Puschel K, Corvalán AH, et al. High-risk HPV infection after five years in a population-based cohort of Chilean women. Infect Agent Cancer [Internet]. 2011 [cited 2021 Jan 25]; 6 (1). Available from: https:// pubmed.ncbi.nlm.nih.gov/22087645/

12. Lagos M, Van De Wyngard V, Poggi H, Cook P, Viviani P, Barriga MI, et al. HPV16/18 genotyping for the triage of HPV positive women in primary cervical cancer screening in Chile. Infect Agent Cancer [Internet].
2015 Nov 23 [cited 2021 Jan 25]; 10 (1). Available from: https://pubmed.ncbi.nlm.nih.gov/26600869/

13. Nanda K, McCrory DC, Myers ER, Bastian LA, Hasselblad V, Hickey JD, et al. Accuracy of the papanicolaou test in screening for and follow-up of cervical cytologic abnormalities: A systematic review. Ann Intern Med. 2000 May 16; 132 (10): 810-9.

14. Ferreccio C, Barriga MI, Lagos M, Ibáñez C, Poggi H, González F, et al. Screening trial of human papillomavirus for early detection of cervical cancer in Santiago, Chile. Int J Cancer [Internet]. 2013 Feb 15 [cited 2021 Jan 25]; 132 (4): 916-23. Available from: http://doi. wiley.com/10.1002/ijc.27662

15. Terrazas S, Ibáñez C, Lagos M, Poggi H, Brañes J, Barriga MI, et al. Human papillomavirus testing in cervical cancer screening at a public health service of santiago, Chile. Rev Med Chil [Internet]. 2015 Jan 1 [cited 2021 Jan 25]; 143 (1): 56-62. Available from: https://pubmed. ncbi.nlm.nih.gov/25860269/

16. Wright TC, Stoler MH, Behrens CM, Sharma A, Zhang G, Wright TL. Primary cervical cancer screening with human papillomavirus: End of study results from the ATHENA study using HPV as the first-line screening test. Gynecol Oncol [Internet]. 2015 Feb 1 [cited 2021 Jan 25]; 136 (2): 189-97. Available from: http://dx.doi. org/10.1016/j.ygyno.2014.11.076

17. Global strategy to accelerate the elimination of cervical cancer as a public health problem [Internet]. [cited 2021 Jan 25]. Available from: https://www.who.int/publications/i/item/9789240014107

18. Almonte M, Murillo R, Sánchez GI, Jerónimo J, Salmerón J, Ferreccio C, et al. Nuevos paradigmas y desafíos en la prevención y control del cáncer de cuello uterino en América Latina. Salud Publica Mex [Internet]. 2010 [cited 2021 Jan 25]; 52 (6): 544-59. Available from: https://pubmed.ncbi.nlm.nih.gov/21271014/

19. Elfström KM, Arnheim-Dahlström L, Von Karsa L, Dillner J. Cervical cancer screening in Europe: Quality assurance and organisation of programmes. Eur J Cancer. 2015 May 1; 51 (8): 950-68.

20. Chrysostomou AC, Stylianou DC, Constantinidou A, Kostrikis LG. Cervical cancer screening programs in Europe: The transition towards HPV vaccination and population-based HPV testing [Internet]. Viruses. MDPI AG 2018 [cited 2020 Sep 1]; 10. Available from: / pmc/articles/PMC6315375/?report=abstract

21. Dijkstra MG, van Zummeren M, Rozendaal L, van Kemenade FJ, Helmerhorst TJM, Snijders PJF, et al. Safety of extending screening intervals beyond five years in cervical screening programmes with testing for high risk human papillomavirus: 14 year follow-up of population 
based randomised cohort in the Netherlands. BMJ [Internet]. 2016 Oct 4 [cited 2020 Sep 1]; 355: i4924. Available from: http://dx.doi.org/10.1136/bmj.i4924

22. Human Papillomavirus and Related Diseases Report EUROPE [Internet]. [cited 2020 Sep 1]. Available from: www.hpvcentre.net

23. Integrating HPV testing in cervical cancer screening program: a manual for program managers [Internet]. [cited 2020 Sep 1]. Available from: https://iris.paho.org/ handle/10665.2/31393

24. Huh WK, Ault KA, Chelmow D, Davey DD, Goulart RA, Garcia FAR, et al. Clinical Commentary Use of primary high-risk human papillomavirus testing for cervical cancer screening: Interim clinical guidance. Gynecol Oncol [Internet]. 2014 [cited 2020 Sep 1]; Available from: http://dx.doi.org/10.1016/j.ygyno.2014.12.022http:// dx.doi.org/10.1016/j.ygyno.2014.12.0220090-8258/

25. Huh WK, Ault KA, Chelmow D, Davey DD, Goulart RA, Garcia FAR, et al. Use of Primary High-Risk Human Papillomavirus Testing for Cervical Cancer Screening. Obstet Gynecol [Internet]. 2015 Feb 6 [cited 2020 Sep 1]; 125 (2): 330-7. Available from: http://journals.lww. com/00006250-201502000-00008

26. Sawaya GF, Sanstead E, Alarid-Escudero F, Smith-Mccune K, Gregorich SE, Silverberg MJ, et al. Estimated Quality of Life and Economic Outcomes Associated with 12 Cervical Cancer Screening Strategies: A Cost-effectiveness Analysis. JAMA Intern Med [Internet]. 2019 Jul 1 [cited 2020 Sep 1]; 179 (7): 867-78. Available from: https://jamanetwork.com/

27. Hall MT, Simms KT, Lew J Bin, Smith MA, Brotherton
JM, Saville M, et al. The projected timeframe until cervical cancer elimination in Australia: a modelling study. Lancet Public Heal. 2019 Jan 1; 4 (1): e19-27.

28. Smith M, Hammond I, Saville M. Lessons from the renewal of the national cervical screening program in Australia. Public Heal Res Pract [Internet]. 2019 [cited 2020 Sep 1]; 29 (2). Available from: https://www.phrp. com.au/issues/july-2019-volume-29-issue-2/lessonsfrom-the-renewal-of-the-national-cervical-screeningprogram-in-australia/

29. Ministerio de Salud G de C. Plan Nacional de Cáncer 2018-2028 [Internet]. 2018 [cited 2019 Jan 4]. Available from: https://cdn.digital.gob.cl/filer_public/d3/0a/ d30a1f5e-53d9-4a31-a4fe-e90d8d9a2348/documento_plan_nacional_de_cancer.pdf

30. Washington DC. 62nd Session of the executive committee plan of action for cervical cancer prevention and control 2018-2030 [Internet]. PAHO 2018 [cited 2021 Jan 25]. Available from: https://iris.paho.org/handle/10665.2/49490

31. Ferreccio C, Barriga MI, Lagos M, Ibáñez C, Poggi H, González F, et al. Screening trial of human papillomavirus for early detection of cervical cancer in Santiago, Chile. Int J Cancer [Internet]. 2012 Feb 15 [cited 2021 May 24]; 132 (4): 916-23. Available from: https://onlinelibrary.wiley.com/doi/full/10.1002/ijc.27662

32. Ferreccio C. New strategies for the prevention and control of cervical cancer in Chile. Salud Publica Mex [Internet]. 2018 Nov 1 [cited 2021 Jan 25]; 60 (6): 713-21. Available from: https://pubmed.ncbi.nlm.nih. gov/30699276/ 